Cerebrovasc Dis 2006;21:142-144

DOI: 10.1159/000090450

\section{A Giant Intracavernous Carotid Artery Aneurysm Embedded in Pituitary Macroadenoma Presenting with Pituitary Apoplexy}

Chi Cheng Chuang ${ }^{\mathrm{a}}$, Yao Liang Chen ${ }^{\mathrm{b}}$, Ping Ching Pai ${ }^{\mathrm{c}}$

Department of a Neurosurgery, beuroradiology, and ${ }^{\mathrm{c}}$ Radiation Oncology, Chang Gung Memorial Hospital, Chang Gung University, Linkou, Taiwan, ROC

\section{Introduction}

The location of an aneurysm associated with a pituitary adenoma is quite variable. It has been described as near, contiguous or embedded in a pituitary adenoma. Management of coexistence of an intracavernous aneurysm and pituitary macroadenoma with apoplexy can be achieved by minimal invasive procedures, such as endovascular embolization for aneurysm and transsphenoidal surgery for tumor resection. However, another approach is needed in situations of an apoplectic event with severe visual compromise where endovascular treatment failed. Proximal trapping of the internal carotid artery (ICA) cannot ensure the complete obliteration of the aneurysm. Thus, craniotomy for tumor resection and trapping of the ICA immediately distal to the aneurysm were performed simultaneously. We discussed this treatment method and reviewed the literature $[1,2]$.

\section{Case Report}

A 60 -year-old man presented with slow decreasing visual acuity for 3 months. Sudden deterioration in the right side vision, accompanied by severe headache and vomiting, was noted 1 day prior to admission. Physical examination revealed mild fever, neck stiffness and blunting of consciousness. Emergent brain CT showed a bright mass within the sella which was thought compatible with pituitary apoplexy. The mass extended into the right cavernous sinus with bony destruction. A brain MRI was arranged before emergent optic nerve decompression via the transsphenoidal route. The MRI of the brain revealed an intracavernous carotid aneurysm with a signal void embedded in pituitary macroadenoma. The tumor was located in the sellar region with marked suprasellar extension (fig. 1). High-dose glucocorticoids were administered after diagnosis, and cerebral angiography for embolization of the aneurysm was performed. Due to difficulties in identifying the neck of the aneurysm, we checked the collateral circulation by the balloon occlusion test. An angiogram showed good collateral blood supply through both the anterior and posterior communicating arteries. The patient tolerated the occlusion test well. Trapping was not done, but proximal occlusion of the right ICA was performed with detachable
Fig. 1. A saccular flow void signal on an axial $\mathrm{T}_{2}$-weighted image (arrow in $\mathbf{A}$ ) and sagittal $\mathrm{T}_{1}$-weighted images (arrow in $\mathbf{C}$ ) locates within the sellar mass, which is enhanced with gadolinium (arrowheads in $\mathbf{B}$, D), suggesting an aneurysm. The suprasellar component exhibits eccentric growth with heterogenous enhancement (double arrowheads in C, D), presumably a coexistent pituitary macroadenoma.
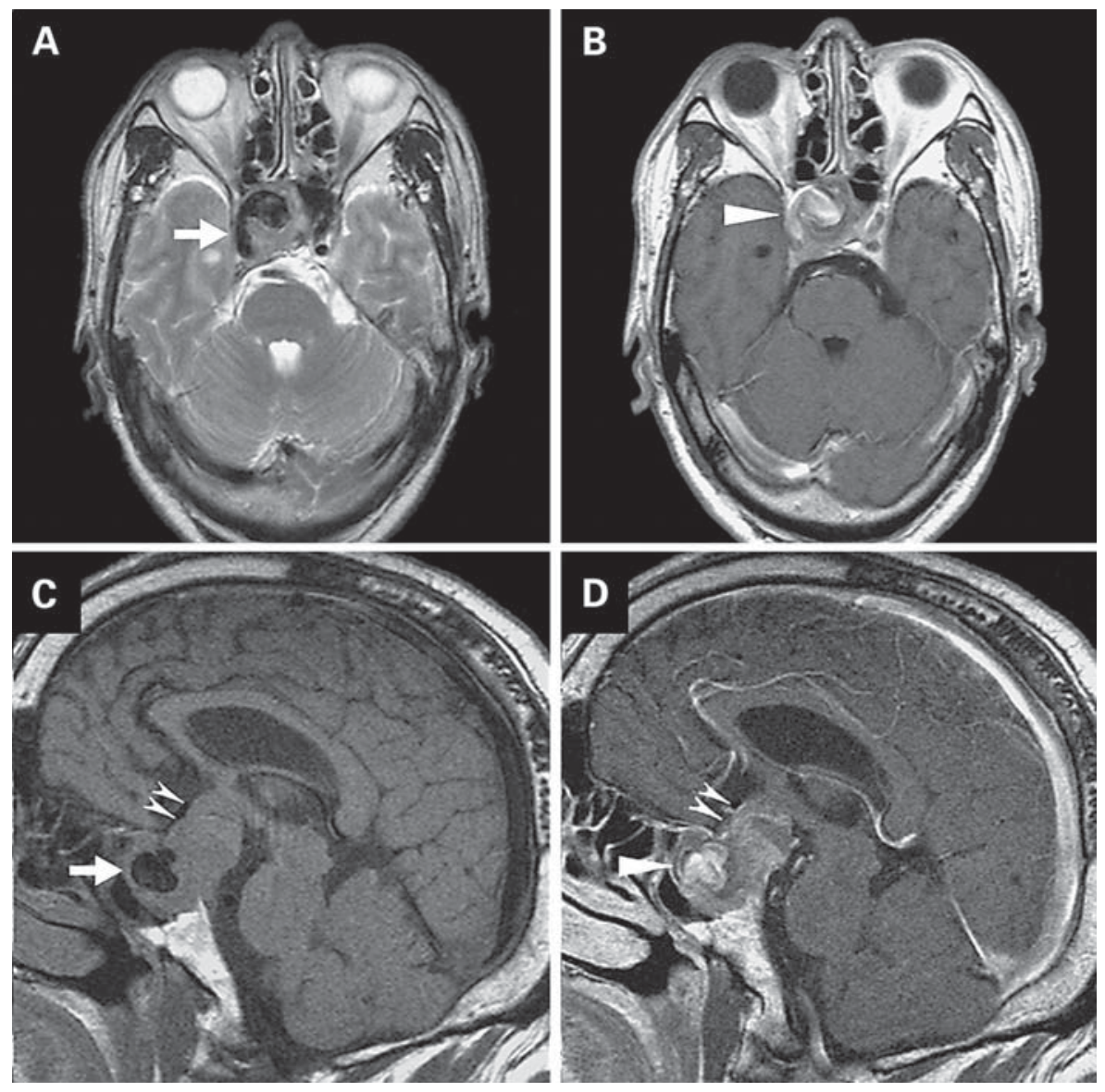
coils (fig. 2). No new neurological deficit developed after this procedure. The next day, the patient underwent a right frontotemporal craniotomy, occluding the right ICA proximal to the right posterior communicating artery with an aneurysm clip and evacuating the tumor and hematoma. During resection procedure, no clear demarcation between adenoma and blood clot was found. The hematoma extended from sella to suprasella and was surrounded by tumor tissue. A mixture of dark red, fragile adenomatous substance and large blood clot within the tumor capsule was evacuated. It might be the evidence for a ruptured aneurysm embedded in adenoma. The histopathologic examination revealed a nonfunctional adenoma with apoplexy.

The course was uneventful after surgery. Partial recovery of the right eye vision was noted. The patient received glucocorticoid and thyroid hormone replacement therapy during the follow-up period. A small nonenhanced granulation tissue was located in the right cavernous sinus on the follow-up MRI 3 years later (fig. 3).
Fig. 2. A, B Right ICA angiogram: a giant aneurysm in the right cavernous sinus and wide base of the neck. C, D Good collateral circulation supply via anterior and posterior communicating arteries. Total occlusion of the aneurysm and right ICA were done with detachable coils (arrows).

Fig. 3A, B. Decompression for optic nerve and pituitary gland was well achieved after craniotomy, removal of the aneurysm and tumor, and the only residual was granulation tissue in the right cavernous sinus.
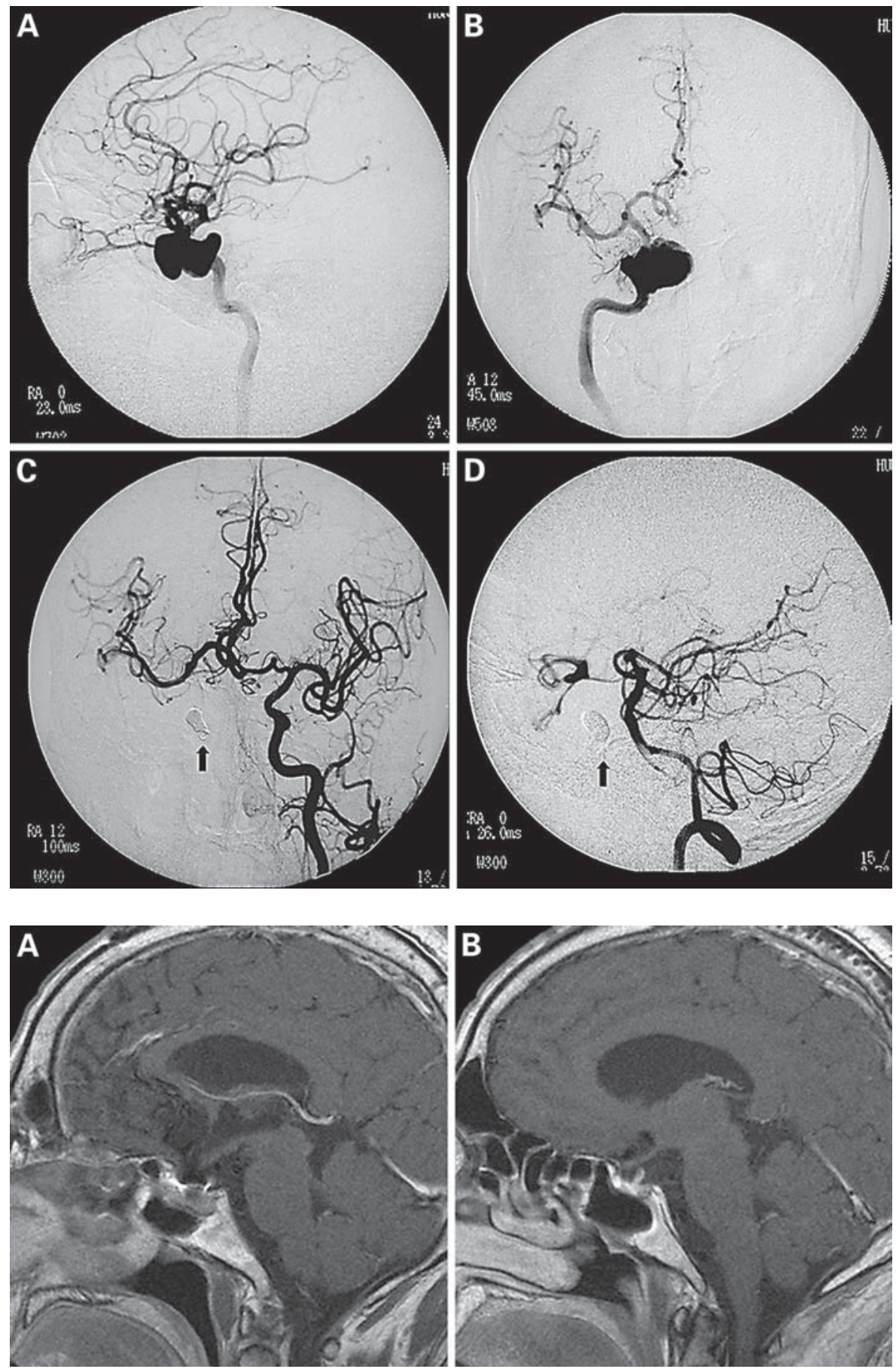


\section{Discussion}

Incidence and Mechanism. The coincidence of cerebral aneurysms and pituitary apoplexy has been reported to be as high as $12.3 \%$ [3]. Most cases of associated aneurysm were found in the anterior circulation [4]. Acqui et al. [5], Jakubowski and Kendall [6], Pia et al. [7] and Wakai et al. [8] reported cases that emphasize a higher incidence of aneurysms in patients with pituitary adenomas, especially growth hormone-secreting tumors, than in the general population. Several mechanisms of aneurysm formation associated with pituitary adenoma were proposed, such as local circulatory stress, endocrine effect, mechanical effect and direct invasion. It is possible that one or more different mechanisms play a role in different aneurysm locations.

On the other hand, in a recent review by Pant et al. [4], the authors conducted a large-scale study and found no association between hormone secretion, size or the invasive nature of the tumors. The occurrence of an intracranial aneurysm with pituitary adenoma was merely a chance finding, and the incidence of aneurysms in patients with pituitary adenomas was no greater than in the general population.

Only a few cases of unruptured intrasellar aneurysms contiguous to pituitary adenomas have been described [8,9], and to our knowledge, only one case of pituitary apoplexy caused by aneurysmal bleeding into a pituitary adenoma has been reported [2]. In that case, intraoperative massive bleeding was noted and postoperative angiography revealed an intracavernous carotid artery aneurysm.

Treatment. The definitive treatment for pituitary apoplexy is surgery for decompression of constricted cavernous and/or suprasellar structures [10]. Significant visual compromise or diminished levels of consciousness are clear indications for an early operation. Surgery offers a means for direct and immediate decompression and optimizes the recovery of the endocrine and visual function. A transsphenoidal approach is the first choice in most instances. Medical treatment with dopamine agonists in conjunction with steroid administration is used for some cases of prolactinomas presenting with isolated and stable meningismus and/or ophthalmoplegia [10].

Most asymptomatic intracavernous ICA aneurysms do not require surgical treatment. Because the morbidity and mortality rates associated with surgical treatment are high, surgery should be reserved for cases with asymptomatic aneurysms that are associated with a considerable risk of subarachnoid or intracranial hemorrhage. Methods of direct clipping, trapping and bypass, and wrapping are used in different surgical conditions. In trapping cases, the ICA was occluded in the petrous region or neck, as well as intracranially immediately distal to the aneurysm. Proximal occlusion was likely to be less effective because of large collateral back flow to the aneurysm via the ophthalmic artery [11]. Thus, craniotomy for proximal ICA ligation is necessary for proximal trapping cases.

Intracavernous aneurysms associated with adenomas can be treated by both endovascular and microsurgical techniques, simultaneously or separately $[9,12]$. The embolization method accomplishes better treatment results than the surgical approach for aneurysms located in a difficult region. Facing this kind of disease, many procedures should be prepared, including the course of embolization, a balloon occlusion test for trapping, and extracranialintracranial bypass surgery for insufficiency of collateral circulation. The choice of craniotomy or transsphenoidal surgery is based on the size and suprasellar extension of the tumor and how the aneurysm was treated.

\section{References}

1 Imamura J, Okuzono T, Okuzono Y: Fatal epistaxis caused by rupture of an intratumoral aneurysm enclosed by a large prolactinoma: case report. Neurol Med Chir (Tokyo) 1998;38:654-656.

2 Suzuki H, Muramatsu M, Murao K, Kawaguchi K, Shimizu T: Pituitary apoplexy caused by ruptured internal carotid aneurysm. Stroke 2001;32: $567-569$.

3 Fraioli B, Esposito V, Palma L, Cantore G: Hemorrhagic pituitary adenomas: clinicopathological features and surgical treatment. Neurosurgery 1990;27:741-748.

4 Pant B, Arita K, Kurisu K, Tominaga A, Eguchi K, Uozumi T: Incidence of intracranial aneurysm associated with pituitary adenoma. Neurosurg Rev 1997;20:13-17.

5 Acqui M, Ferrante L, Fraioli B, Cosentino F, Fortuna A, Mastronardi L: Association between intracranial aneurysms and pituitary adenomas. Aetiopathogenetic hypotheses. Neurochirurgia (Stuttg) 1987;30:177-181.

6 Jakubowski J, Kendall B: Coincidental aneurysms with tumors of pituitary origin. J Neurol Neurosurg Psychiatry 1978;41:972-979.

7 Pia HW, Obrador S, Martin JG: Association of brain tumors and arterial intracranial aneurysms. Acta Neurochir (Wien) 1972;27:189-204.

8 Wakai S, Fukushima T, Furrihata T, Sano K: Association of cerebral aneurysm with pituitary adenoma. Surg Neurol 1979;12:503-507.

9 Sade B, Mohr G, Tampieri D, Rizzo A: Intrasellar aneurysm and a growth hormone-secreting pituitary macroadenoma. Case report. J Neurosurg 2004; 100:557-559.

10 Verrees M, Arafah BM, Selman WR: Pituitary tumor apoplexy: characteristics, treatment, and outcomes. Neurosurg Focus 2004;16:E6.

11 Kikuta K, Miyamoto S, Satow T, Kataoka H, Hashimoto N: Large paraclinoid aneurysm with a calcified neck treated by tailored multimodality procedures. Neurol Med Chir (Tokyo) 2005;45:196-200.

Ping Ching Pai, MD, Department of Radiation Oncology Chang Gung Memorial Hospital, 5, Fu-Shin Street, Kwei-Shan Hsiang Taoyuan Hsien, 333, Taiwan (ROC)

Tel. +886 3 3281200, ext. 2600, 2604, Fax +886 33280797

E-Mail ccc2915@adm.cgmh.org.tw 